\title{
Development of one-axis active controlled bearingless motor working at extremely low temperature
}

\author{
Hirohisa KATO*, Mochimitsu KOMORI*, Ken-ichi ASAMI* and Nobuo SAKAI* \\ ${ }^{*}$ Kyushu Institute of Technology \\ 1-1 Sensui, Tobata, Kitakyushu, Fukuoka 804-8550, Japan \\ E-mail: komori_mk@yahoo.co.jp
}

Received: 26 June 2019; Revised: 15 August 2019; Accepted: 24 September 2019

\begin{abstract}
This paper discusses the manufactured one-axis controlled bearingless motor and its experimental results in liquid nitrogen. The bearingless motor is an axial type bearingless motor. It generates a force in the axial direction to control the axial displacement of the rotor and a rotation torque for the motor. The axial displacement is actively controlled using a PID controller, and the rotation speed of the rotor is also actively controlled using a PI controller. The other degrees of freedom are passively supported by the repulsive force of the permanent magnet bearings. The axial type bearingless motor consists of a stator, a rotor, three displacement sensors, and three Hall sensors. Displacement sensors and Hall sensors are used to measure the axial displacement and rotation angle of the rotor. The stator has six salient poles, and the rotor has four poles with four permanent magnets. The air gap between the stator and rotor is $1.5 \mathrm{~mm}$ at the center position. The axial type bearingless motor and permanent magnet bearings are set in liquid nitrogen in the experiment. The experimental results show an impulse response at $0 \mathrm{rpm}$, the relationship between the displacement of the rotor and rotation speed, the relationship between the driving current and the rotation speeds, and a step response from $500 \mathrm{rpm}$ to $1,000 \mathrm{rpm}$ in the rotation speed. From experimental results, it is confirmed the stable levitation and rotation of the bearingless motor at liquid nitrogen temperature.
\end{abstract}

Keywords : Magnetic bearing, Bearingless motor, Actuator, Rotary machinery, Centrifugal pump

\section{Introduction}

Liquefied natural gas and liquid hydrogen are expected as alternative energy for petroleum (Ministry of Economy, Trade and Industry). These liquids require a pump with a dedicated bearing for low temperature liquids below $100 \mathrm{~K}$. Ceramic ball bearings are used in conventional cryogenic centrifugal pumps but frequent maintenance requirements due to bearing wear problems. To solve this problem, a cryogenic centrifugal pump using a magnetic bearing has been developed (Komori, et al., 2004, 2017) (Okada, et al., 2014). They solve the problem by supporting the rotor in a non-contact support using magnetic bearings or superconductors. A frameless eddy current sensor has also been developed for cryogenic displacement measurement, which are indispensable for magnetic bearings in extremely low temperature (Wang, et al., 2010).

However, magnetic bearings in cryogenic centrifugal pumps are not widely adopted due to the problem of high cost of the magnetic bearings (Okada, et al., 1995). Sensors, controllers, inverters, etc. are required to use magnetic bearings. In response to the cost problem, one-axis controlled bearingless motors or self-bearing motors have been developed (Ueno and Arakawa, 2014) (Ueno, et al., 2000) (Sugimoto, et al., 2016). This reduces the cost of the magnetic bearing by reducing the number of sensors, controllers, and inverters by setting the controlled axis only in the axial direction. Some rotary machines for extremely low temperatures using magnetic bearings have been reported (Komori, et al., 2017) (Okada, et al., 2014), but there are no examples of study on such rotary machines using bearingless motors.

In this paper, we show the results of levitation and rotation experiments in liquid nitrogen (77 K) with fabricating one-axis controlled bearingless motor which is axial type bearingless motor (ABM). The axial direction is actively 
controls using $\mathrm{ABM}$ and PID controller, and the other four degrees of freedom are passively supported by permanent magnet bearings (PMBs) using the repulsive force of permanent magnets. It confirmed that the ABM is levitated and rotated from $100 \mathrm{rpm}$ to $1,900 \mathrm{rpm}$ in liquid nitrogen.

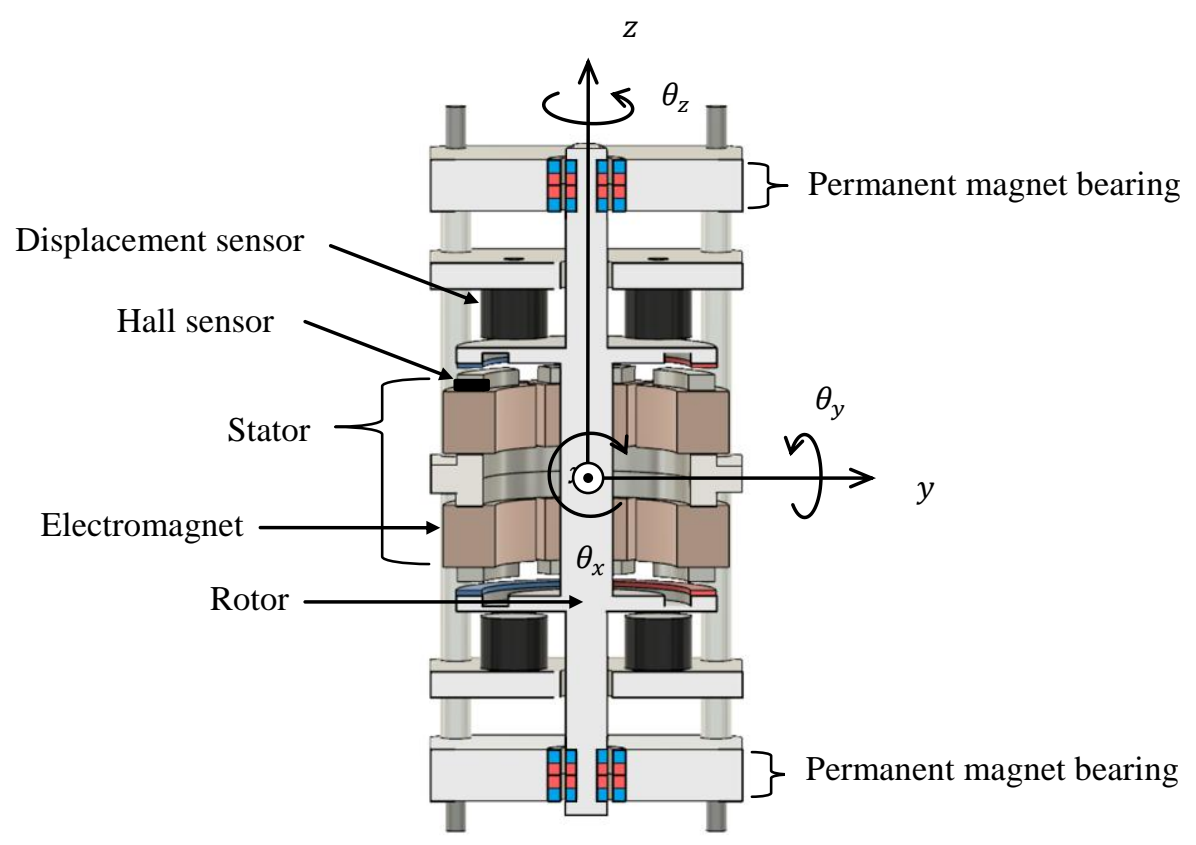

Fig. 1 Structure of axial type bearingless motor which consists of a stator, a rotor, displacement sensors, and Hall sensors. The PMBs passively control displacements and rotations in the $x, y, \theta_{x}$, and $\theta_{y}$ directions. The coordinate point is the center position of the stator.

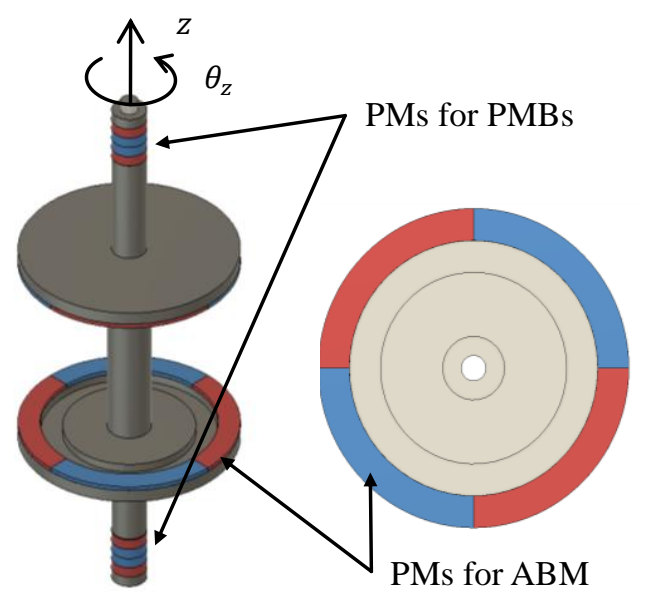

(a) Rotor

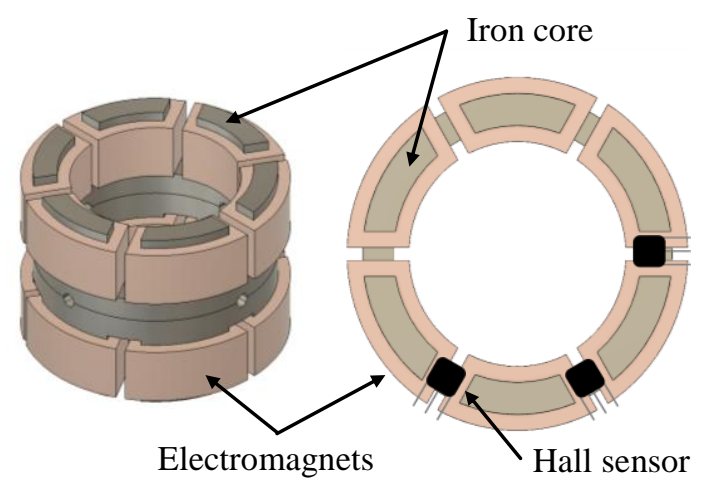

(b) Stator

Fig. 2 Schematic drawing and cross-section of (a) the rotor and (b) the stator. The rotor consists of permanent magnet for PMBs and ABM, rotor core and shaft. The stator consists of Electromagnets and a stator core.

\section{Structure}

\subsection{Bearingless motor}

The structure of ABM is shown in Fig. 1. It consists of a stator, a rotor, three displacement sensors, and three Hall sensors. The rotor is levitated using the ABM and two permanent magnet bearings (PMBs) and rotates around the $z$ axis using the ABM. The ABM generates a force in the $z$ direction and a rotating torque in the $\theta_{z}$ direction of rotation to the rotor. Displacement sensors and Hall sensors are set to measure the axial displacement and rotation angle of the rotor. The displacement in the $z$ direction is actively controlled using PID controller. The rotation speed in the $\theta_{z}$ direction is also actively controlled using PI controller. The $x$ and $y$ displacement and the $\theta_{x}$ and $\theta_{y}$ rotation angle are passively 
supported by the radial repulsive force in the PMBs. The permanent magnet forces in the ABM and PMBs are balanced when the rotor is in the center position.

Figure 2 shows a schematic drawing and cross-section of the rotor and the stator. Eight permanent magnets (PMs) are set to be 4-pole for ABM as shown in Fig. 2 (a). Four PMs are set on both ends of the rotor for PMBs. The axis of rotation of the rotor is in the $z$ direction. The stator consists of an iron core and twelve electromagnets of a concentrated winding as shown in Fig. 2(b). Three Hall sensors are set to detect the rotation angle in the $\theta_{z}$. at 60 degree intervals between electromagnets. The stator has six salient poles on one side, and the rotor has four poles with four PMs.

Parameters of the ABM are shown in Table 1. The material of the rotor and stator core is pure iron. The stator is 50 $\mathrm{mm}$ in outer diameter, $40 \mathrm{~mm}$ in inner diameter and $40 \mathrm{~mm}$ in height. The gap between slots of the stator is $7 \mathrm{~mm}$ in width and $15 \mathrm{~mm}$ in depth. The rotor is $50 \mathrm{~mm}$ in outer diameter and $126 \mathrm{~mm}$ in height. The air gap is $1.5 \mathrm{~mm}$ between $\mathrm{PM}$ of $\mathrm{ABM}$ and top of electromagnets. The PM of ABM is NdFeB (N40), $25 \mathrm{~mm}$ in outer radius, $20 \mathrm{~mm}$ in inner radius, $1.0 \mathrm{~mm}$ in height, 90 degrees in central angle. Electromagnet is $0.3 \mathrm{~mm}$ in wire diameter, 250 turns. Detailed dimensions of the rotor and stator are shown in Fig. 3 and Fig. 4.

Table 1 Parameters of the ABM

\begin{tabular}{cc}
\hline \multicolumn{2}{c}{ Rotor and Stator core } \\
\hline Material & Pure iron \\
Stator core & $\varnothing 50 \times \varnothing 40 \times 40 \mathrm{~mm}$ \\
Rotor core & $\emptyset 50 \times 3.0 \mathrm{~mm}$ \\
Air gap & $1.5 \mathrm{~mm}$ \\
\hline
\end{tabular}

\begin{tabular}{cc}
\hline & PM of ABM and electromagnet \\
\hline PM & NdFeB $(\mathrm{N} 40)$ \\
Size & R $25 \mathrm{~mm} \times \mathrm{r} 20 \mathrm{~mm} \times 1.0 \mathrm{~mm} \times 90^{\circ}$ \\
Wire & $\emptyset 0.3 \mathrm{~mm}$ \\
Turns & 250 \\
\hline
\end{tabular}

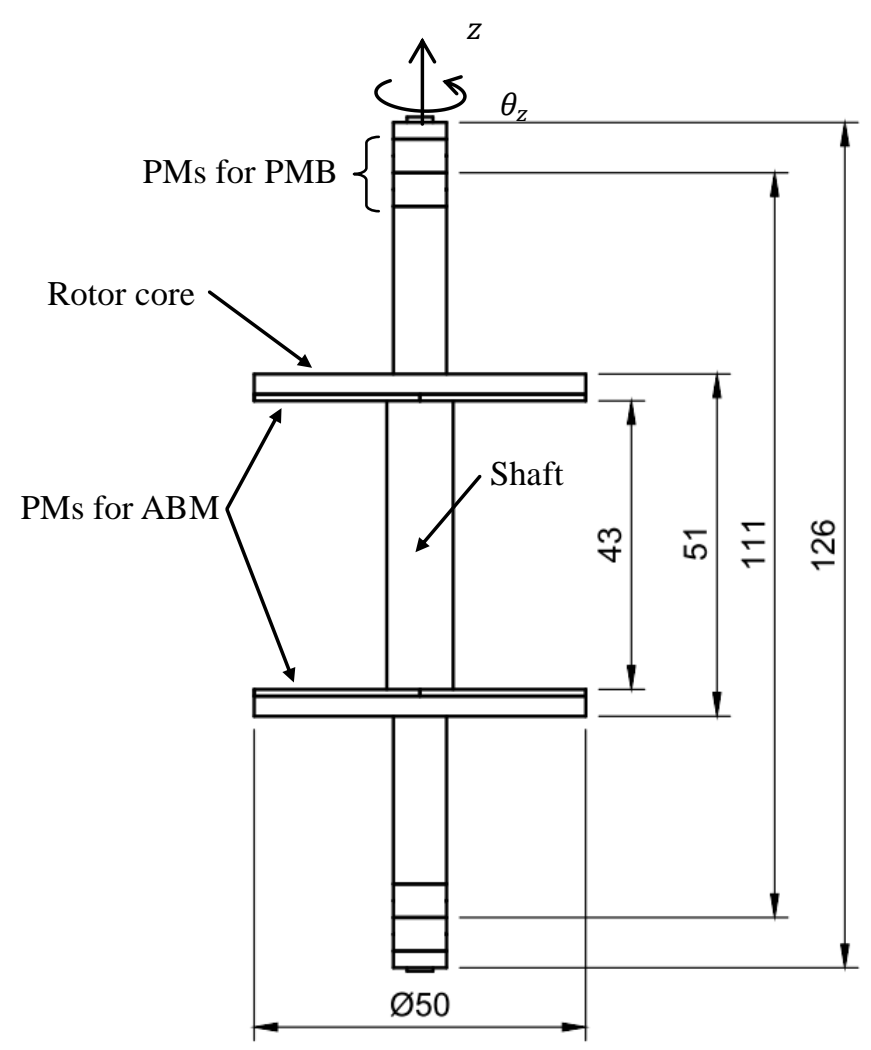

Fig. 3 Front plan view of the rotor. The rotor is $50 \mathrm{~mm}$ in outer diameter and $126 \mathrm{~mm}$ in height. The center position of $\mathrm{PMB}$ is $55.5 \mathrm{~mm}$ in the $\mathrm{z}$ direction from the center position of the rotor.

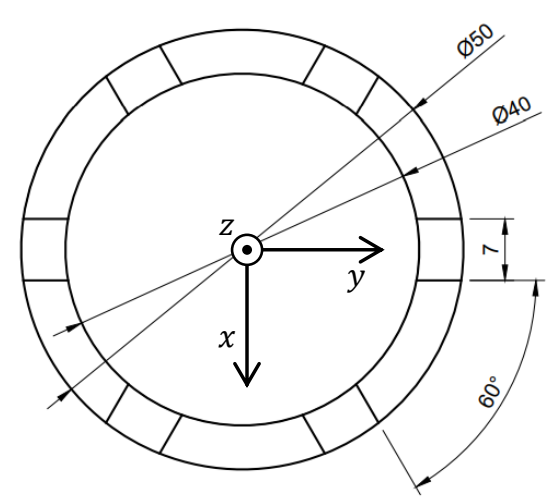

(a) Top plan view

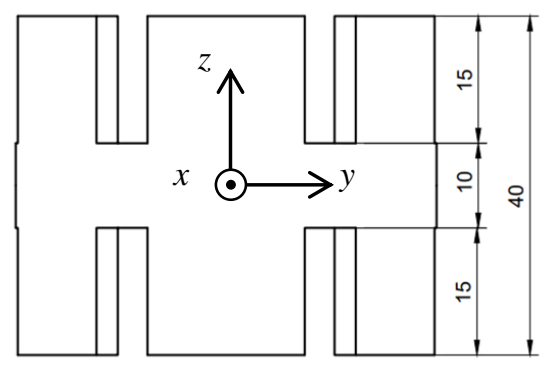

(b) Front plan view

Fig. 4 Drawings of the stator. (a) and (b) are Top plane view and Front plan view of the stator. The stator is $50 \mathrm{~mm}$ in outer diameter, $40 \mathrm{~mm}$ in inner diameter and $40 \mathrm{~mm}$ in height. 

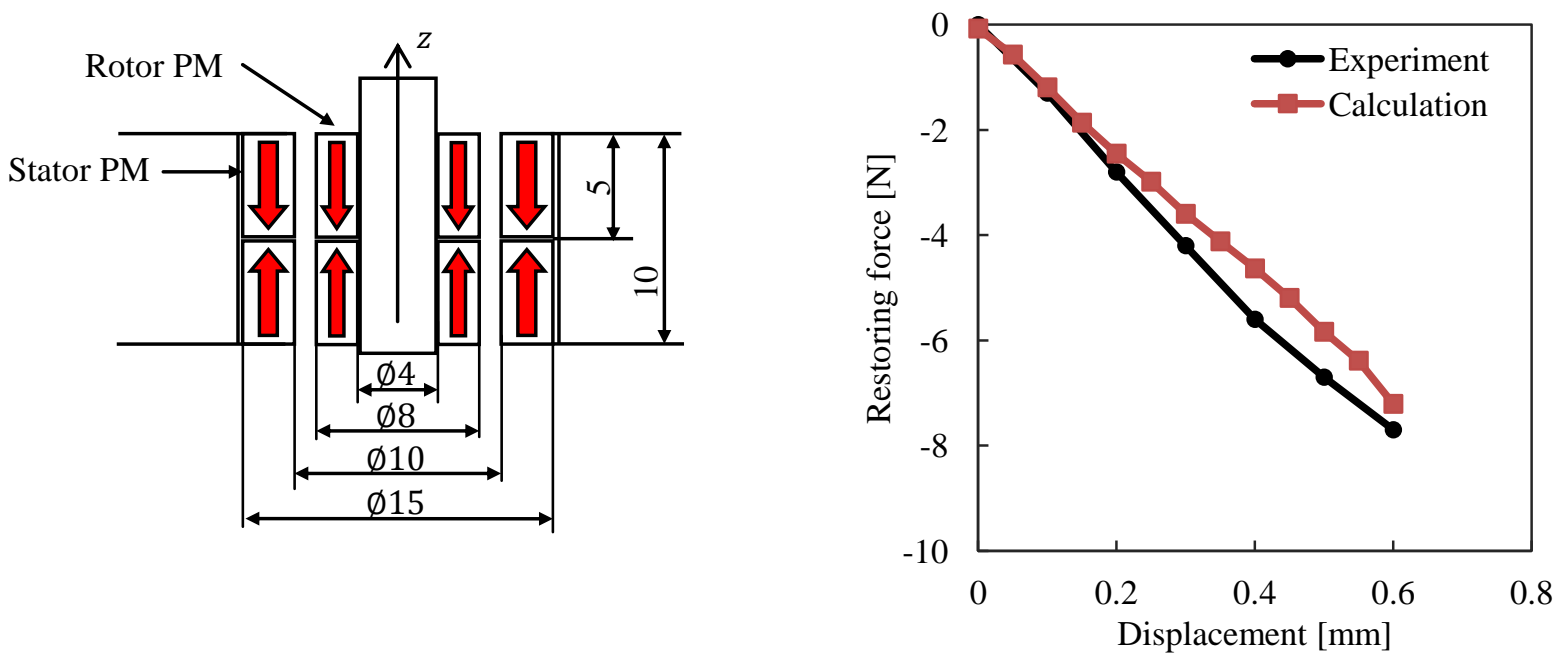

Fig. 5 Schematic of PMs for PMB. It consists of 4 ring-shaped PMs: 2 PMs attached to the stator and 2 PMs attached to the rotor. Arrows indicate the directions in which the PMs are magnetized.

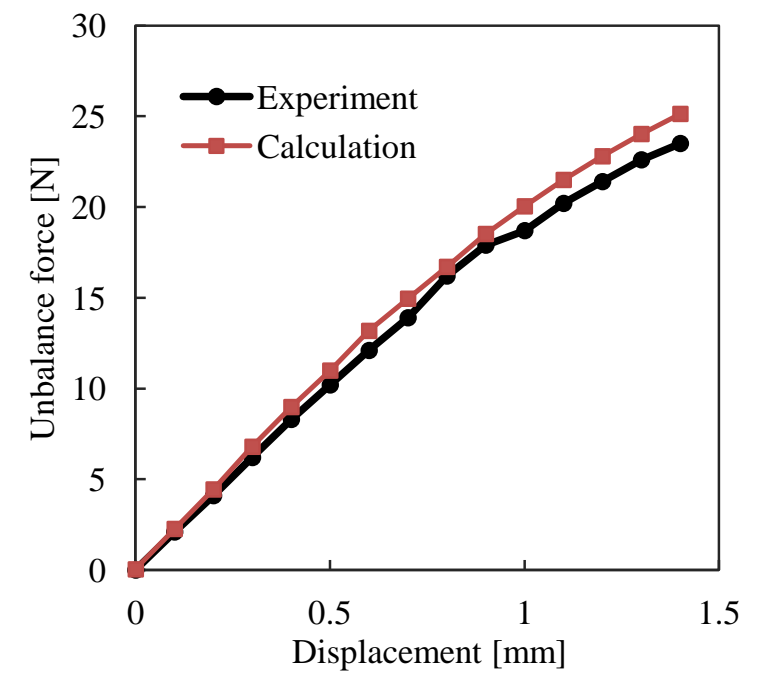

Table 2 Parameters of the PMB

\begin{tabular}{c|c}
\hline Item & Value, Material \\
\hline Material & NdFeB $(\mathrm{N} 40)$ \\
Rotor PM & $\emptyset 8.0 \times \emptyset 4.0 \times 5.0 \mathrm{~mm}$ \\
Stator PM & $\varnothing 15 \times \varnothing 10 \times 5.0 \mathrm{~mm}$ \\
Air gap & $1.0 \mathrm{~mm}$
\end{tabular}

Fig. 7 Relationship between the displacement of the rotor in the axial direction and the unbalanced force of the PMB in the axial direction.

\subsection{FEM analysis and experimental result of $P M B$}

PMB consists of 4 ring-shaped PMs: 2 PMs attached to the stator and 2 PMs attached to the rotor. Since these PMs are magnetized in the direction of the arrow in Fig. 5, the repulsive force of the PMs is generated in the radial and axial directions. JMAG Designer version 10 was used for FEM analysis. A calculated result and an experimental result of the repulsive force are shown in Fig. 6 and Fig. 7. Figure 6 shows the repulsive force in the radial direction. The calculated result shows that the repulsive force is $11.7 \mathrm{~N} / \mathrm{mm}$, and the experimental result is $13.1 \mathrm{~N} / \mathrm{mm}$. This repulsive force is the radial restoring force of the rotor. Figure 7 shows the axial repulsive force. The calculated result shows that the repulsive force is $19.5 \mathrm{~N} / \mathrm{mm}$, and the experimental result is $18.7 \mathrm{~N} / \mathrm{mm}$. This repulsive force is the unbalanced force of the rotor.

Table 2 shows the PMB parameters. The material is $\mathrm{NdFeB}$, and the rotor $\mathrm{PM}$ is $8.0 \mathrm{~mm}$ in the outer diameter, 4.0 $\mathrm{mm}$ in the inner diameter, and $5.0 \mathrm{~mm}$ in the height. The stator PM is $15 \mathrm{~mm}$ in the outer diameter, $10 \mathrm{~mm}$ in the inner diameter, and $5.0 \mathrm{~mm}$ in the height. The air gap between the stator PM and the rotor PM is $1 \mathrm{~mm}$. 


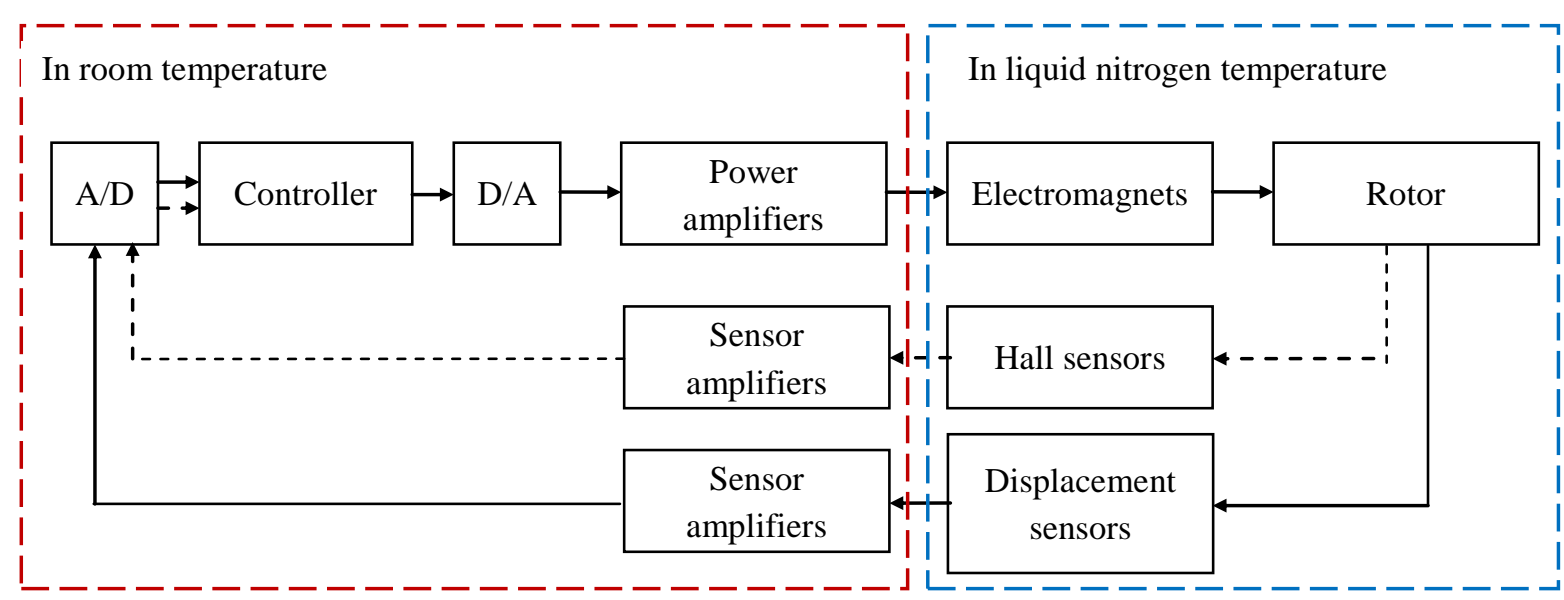

Fig. 8 Block diagram of the control system which consists of electromagnets, a rotor, hall sensors, displacement sensors, power amplifiers, sensor amplifiers, an analog-to-digital converter, a digital-to-analog converter, and a controller. Hall sensors are used to detect the rotation angle and rotation speed of the rotor. Displacement sensors are used to detect the displacement of the rotor in the $z$ direction.

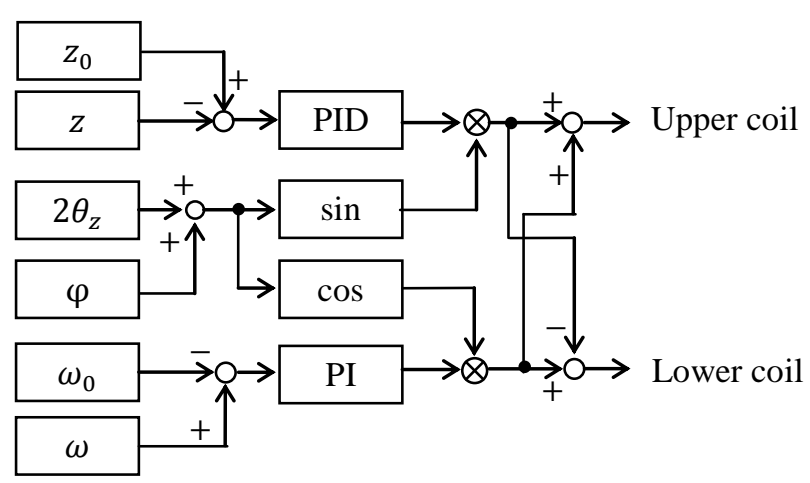

(a) Block diagram of the controller

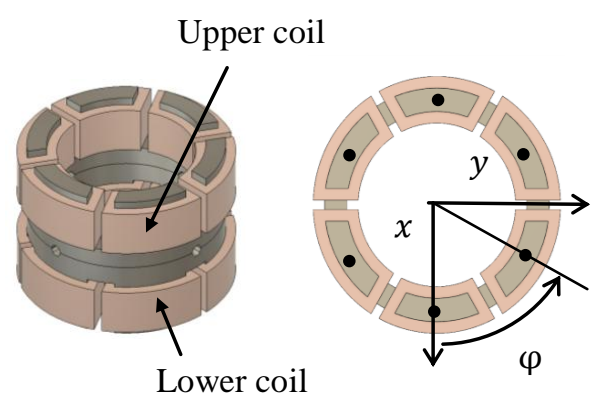

(b) Upper coil, Lower coil and $\varphi$

Fig. 9 Block diagram of the controller. The $z_{0}$ is target value of displacement of the rotor, the $z$ is the displacement in the $z$ direction, the $\theta_{z}$ is the rotation angle of the rotor, the $\varphi$ is the center angle of the electromagnet, the $\omega_{0}$ is target value of rotation speed, the $\omega$ is the rotation speed of the rotor.

\subsection{Control system}

A block diagram of the control system is shown in Fig. 8. It consists of electromagnets, a rotor, hall sensors, displacement sensors, power amplifiers, sensor amplifiers, analog-to-digital converter, digital-to-analog converter, and a controller. Hall sensors are used to detect the rotation angle and rotation speed. Displacement sensors are used to detect the displacement of the rotor in $z$ direction. Electromagnets, the rotor, hall sensors, and displacement sensors are set in liquid nitrogen temperature. Power amplifiers, sensor amplifiers, the analog-to-digital converter, the digital-to-analog converter, and the controller are used at room temperature. The controller was implemented using a digital signal processor (DS1104). The DSP sampling period is set at $100 \mu \mathrm{s}$.

Figure 9 (a) shows a block diagram of the controller. The $z$ is the displacement of the rotor in the $z$ direction, the $\theta_{z}$ is the rotation angle of the rotor, the $\varphi$ is the center angle of the electromagnet (constant for each electromagnet), the $\omega_{0}$ is the reference speed of rotation speed, the $\omega$ is the rotation speed of the rotor. The displacement is controlled using the PID controller, and the rotation speed is controlled using the PI controller. These outputs are distributed to each electromagnet according to $\theta_{z}$ and $\varphi$. The control signal obtained from here is amplified by the power amplifier and flows to the electromagnet. An upper coil, a lower coil, and $\varphi$ are shown in Fig. 9 (b). The $\varphi$ is an angle of 60 
degree intervals from 0 degree to 300 degree.

In extremely low temperatures, the output of displacement sensor changes significantly compared to room temperature because of the reduction of a sensing coil resistance. For this reason, the sensor output is corrected for the change and adjusted to the same characteristics as room temperature in liquid nitrogen. Also, different values are used for PID gain and PI gain in room temperature and liquid nitrogen because of changes in the surface magnetic flux density of PMs and the permeability of the iron core.

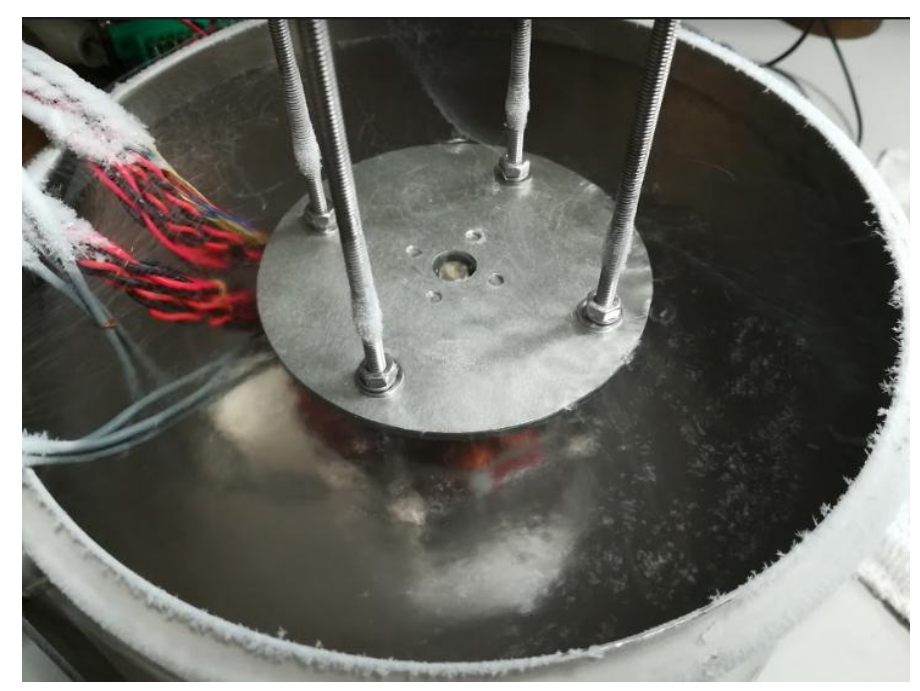

Fig. 10 Photograph of the experimental setup. The ABM is set in liquid nitrogen. Levitation and rotation experiments are carried out using this setting.

Table 3 PID gain of levitation control

\begin{tabular}{c|c}
\hline Item & Value \\
\hline$k_{p}$ & $5.3 \times 10^{3} \mathrm{~A} / \mathrm{m}$ \\
$k_{d}$ & $0.020 \times 10^{3} \mathrm{~A} \cdot \mathrm{s} / \mathrm{m}$ \\
$k_{i}$ & $3.0 \times 10^{3} \mathrm{~A} /(\mathrm{m} \cdot \mathrm{s})$ \\
\hline
\end{tabular}

Table 4 PI gain of rotation speed control

\begin{tabular}{c|c}
\hline Item & Value \\
\hline$k_{p}$ & $1.0 \times 10^{-3} \mathrm{~A} / \mathrm{rpm}$ \\
$k_{i}$ & $1.0 \times 10^{-3} \mathrm{~A} /(\mathrm{rpm} \cdot \mathrm{s})$ \\
\hline
\end{tabular}

Table 5 Physical parameters of the rotor

\begin{tabular}{c|c}
\hline Item & Value \\
\hline$m$ & $0.159 \mathrm{~kg}$ \\
$I_{z}$ & $2.98 \times 10^{-5} \mathrm{~kg} \cdot \mathrm{m}^{2}$ \\
$I_{x}, I_{y}$ & $1.35 \times 10^{-4} \mathrm{~kg} \cdot \mathrm{m}^{2}$ \\
\hline
\end{tabular}

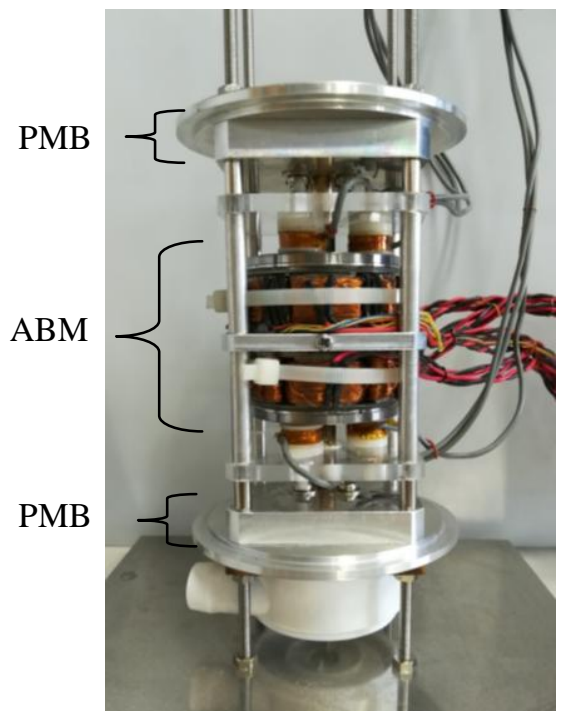

Fig. 11 Photograph of the ABM and PMBs.

\section{Experimental setup}

The experiment was carried out in liquid nitrogen to confirm the stability of the ABM under extremely low temperature. The stator, the rotor, displacement sensors, Hall sensors, and PMBs are set in liquid nitrogen. The photograph of the experiment and the photograph of the ABM and PMBs are shown in Fig. 10 and Fig. 11. Levitation 
and rotation experiments are carried out with this setting. The ABM is set in liquid nitrogen, displacement and Hall sensors also are under liquid nitrogen temperature. The change in temperature affects the output of the displacement sensors and Hall sensors. This change is measured and corrected before the experiment.

In the levitation experiment, the impulse response is measured to confirm the stability of the rotor at $0 \mathrm{rpm}$. In rotation experiments, the rotor vibration and the driving current are measured between $100 \mathrm{rpm}$ and 1,900 rpm. A step response from $500 \mathrm{rpm}$ to $1,000 \mathrm{rpm}$ and the rotor displacement at that time are also measured.

The PID gain of levitation control is shown in Table $3,5.3 \times 10^{3} \mathrm{~A} / \mathrm{m}$ in proportional gain, $0.020 \times 10^{3} \mathrm{~A} \cdot \mathrm{s} / \mathrm{m}$ in differential gain, $3.0 \times 10^{3} \mathrm{~A} /(\mathrm{m} \cdot \mathrm{s})$ in integral gain. The PI gain of rotation speed control is shown in Table 4, $1.0 \times 10^{-3} \mathrm{~A} / \mathrm{rpm}$ in proportional gain, $1.0 \times 10^{-3} \mathrm{~A} /(\mathrm{rpm} \cdot \mathrm{s})$ in integral gain. Physical parameters of the rotor are shown in Table $5,0.159 \mathrm{~kg}$ in mass, $2.98 \times 10^{-5} \mathrm{~kg} \cdot \mathrm{m}^{2}$ in a moment of inertia around $z$ axis at a center point of the rotor, $1.35 \times 10^{-4} \mathrm{~kg} \cdot \mathrm{m}^{2}$ in a moment of inertia around $x$ and $y$ axis at a center point of the rotor.

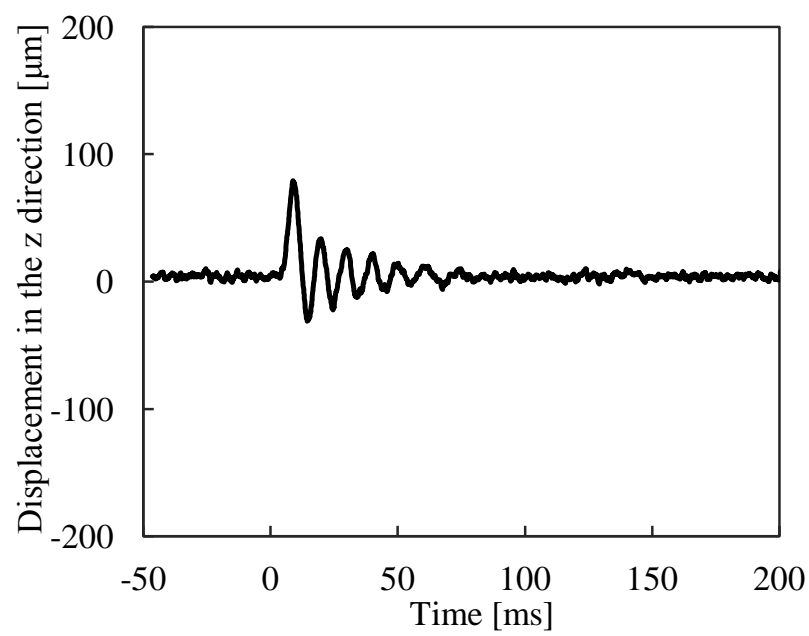

Fig. 12 Relationship between the displacement of the rotor in the $z$ direction and time. The maximum displacement is 84 $\mu \mathrm{m}$ at $8.8 \mathrm{~ms}$. The vibration has converged to the target position within $80 \mathrm{~ms}$. The natural period is about $93 \mathrm{~Hz}$.

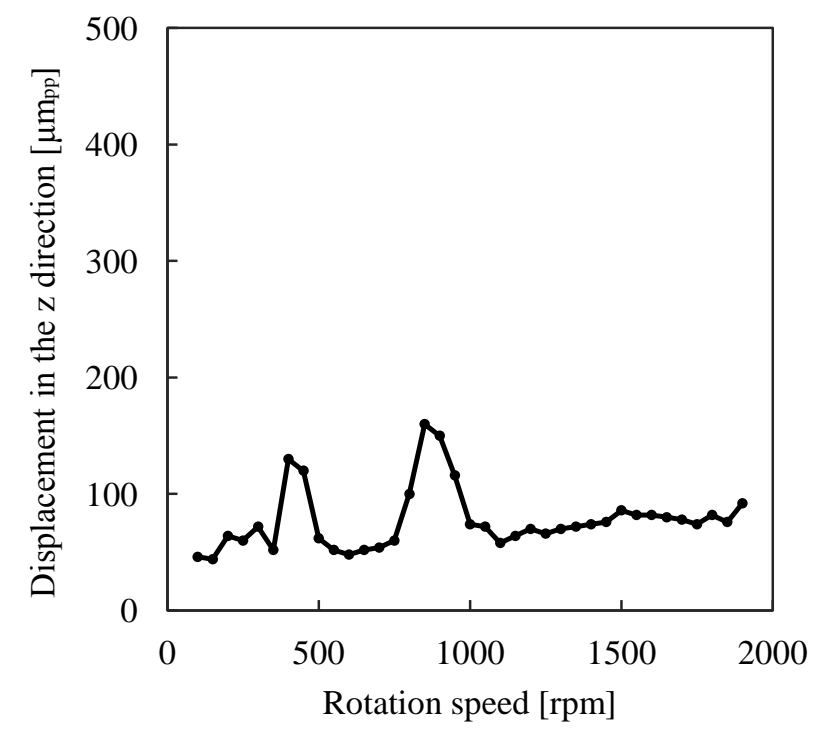

Fig. 13 Relationship between the displacement of the rotor in the $z$ direction and the rotation speed. The displacement is $130 \mu \mathrm{mpp}$ at $400 \mathrm{rpm}, 160$ $\mu \mathrm{mpp}$ at $850 \mathrm{rpm}$.

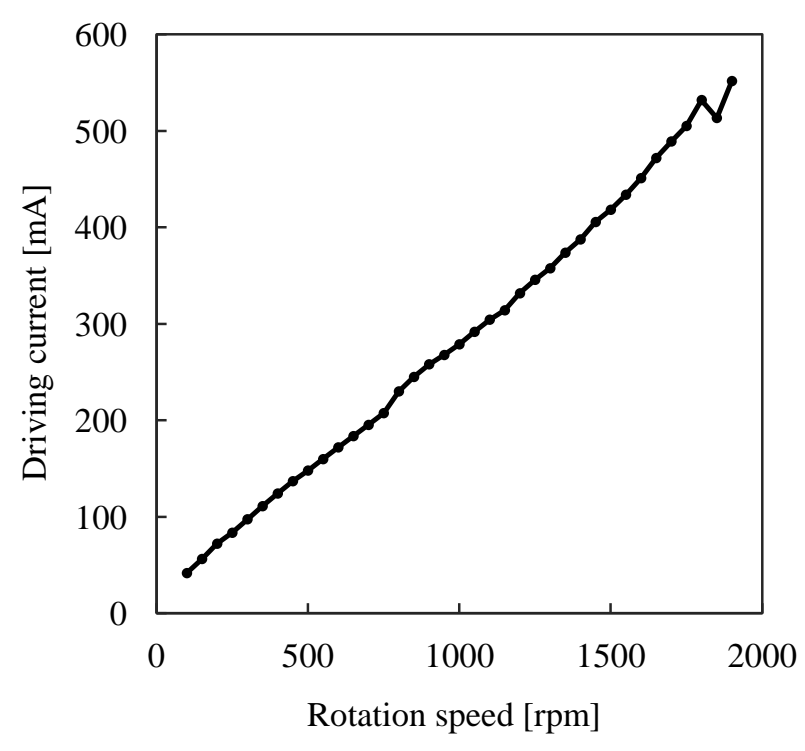

Fig. 14 Relationship between the current of electromagnets and the rotation speed. The driving current is $42 \mathrm{~mA}$ at $100 \mathrm{rpm}$ and 552 $\mathrm{mA}$ at $1,900 \mathrm{rpm}$. 


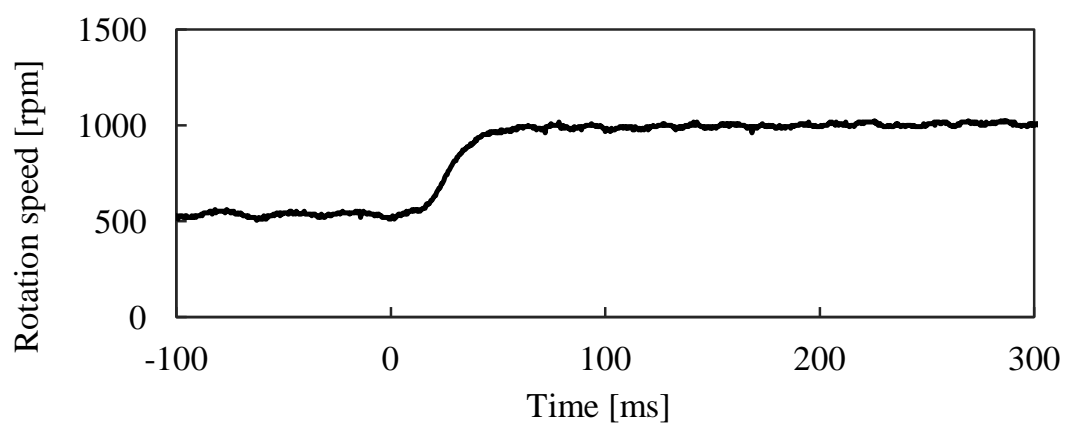

(a) Rotation speed

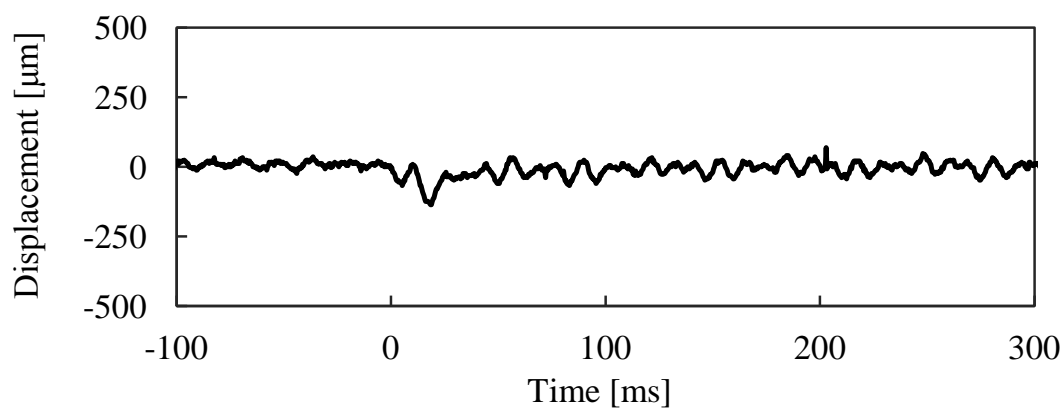

(b) Displacement

Fig. 15 Result of a step response test of the motor. The relationship between the rotation speed and time is shown in (a), and the relationship between the displacement and time is shown in (b). The reference speed of rotation speed is changed from $500 \mathrm{rpm}$ to $1,000 \mathrm{rpm}$ at 0 seconds. The rotation speed has reached the reference speed at $64 \mathrm{~ms}$.

\section{Experimental results}

First, the impulse response is measured at $0 \mathrm{rpm}$ in a state which ABM and PMBs are set in liquid nitrogen. The relationship between the displacement in the $z$ direction and time is shown in Fig. 12. The impulse disturbance is applied at 0 seconds. The maximum displacement is $84 \mu \mathrm{m}$ at $8.8 \mathrm{~ms}$. The vibration has converged to the target position within $80 \mathrm{~ms}$. The natural period is about $93 \mathrm{~Hz}$.

Next, the rotation test is carried out to confirm the stability of ABM in liquid nitrogen. The relationship between the displacement of the rotor in the $z$ direction and the rotation speed is shown in Fig. 13. The rotor is levitated and rotated from $100 \mathrm{rpm}$ to $1,900 \mathrm{rpm}$. The maximum displacement is $160 \mu \mathrm{m}$ peak to peak at $850 \mathrm{rpm}$. The second largest displacement is $130 \mu \mathrm{m}$ peak to peak at $400 \mathrm{rpm}$. These displacements are lower than the air gap of ABM that is $1.5 \mathrm{~mm}$. The stability of the rotor from $100 \mathrm{rpm}$ to $1,900 \mathrm{rpm}$ is confirmed. The cause of peaks at $400 \mathrm{rpm}$ and $850 \mathrm{rpm}$ is due to a cogging torque of the ABM because the rotation speed is considerably lower than the natural period of the rotor.

Figure 14 shows the relationship between the current of electromagnets and the rotation speed. The relationship is clearly linear. The minimum driving current is $42 \mathrm{~mA}$ at $100 \mathrm{rpm}$. The maximum driving current is $552 \mathrm{~mA}$ at 1,900 $\mathrm{rpm}$. The relationship between rotation speed and driving current is $0.277 \mathrm{~mA} / \mathrm{rpm}$. The cause of the increase in drive current around $850 \mathrm{rpm}$ is considered to be due to the energy lost by the vibration of the rotor.

Figure 15 shows the result of a step response in rotation speed. The reference speed of rotation speed is changed from $500 \mathrm{rpm}$ to $1,000 \mathrm{rpm}$ at 0 seconds. The rotation speed has reached the reference speed at 64 ms. The maximum displacement was $136 \mu \mathrm{m}$ at $19 \mathrm{~ms}$. It is confirmed that the rotation speed is rising without contacting the stator while the rotor is accelerating and that the increase in the current for the rotation speed affects the stability of the displacement of the rotor. 


\section{Conclusion}

The one-axis active controlled bearingless motor was developed, and the stability of rotor displacement in liquid nitrogen was measure. The ABM generates a force in the axial direction to control the axial displacement of the rotor and a rotation torque for the motor. The axial displacement is actively controlled using a PID controller, and the rotation speed of the rotor is also actively controlled using a PI controller. The other degrees of freedom are passively supported by the repulsive force of the permanent magnet bearings. From the experiments, it is confirmed that the $\mathrm{ABM}$ is levitated and rotated in liquid nitrogen. The rotor is rotated from $100 \mathrm{rpm}$ to 1,900 rpm. The maximum displacement in the $\mathrm{z}$ direction is $160 \mu \mathrm{m}$ peak to peak at $850 \mathrm{rpm}$. The driving current is $552 \mathrm{~mA}$ at $1,900 \mathrm{rpm}$. The rotation speed has reached from $500 \mathrm{rpm}$ to $1,000 \mathrm{rpm}$ within $64 \mathrm{~ms}$.

Sensor output and shaft rigidity change due to changes in physical properties at extremely low temperatures, but the bearingless motor was operated stably in liquid nitrogen by adjusting the sensor output and PID gain. From experimental results, the vibration of the rotating rotor was sufficiently small for the air gap. The drive current was linear with its rotation speed of the rotor. The step response from $500 \mathrm{rpm}$ to 1,000 rpm reached 1,000 rpm at $64 \mathrm{~ms}$. At that time, the rotor was displaced due to the influence of the drive current. The displacement immediately recovered to the center position and the rotor was levitated and rotated stably.

\section{References}

Komori, M. and Uchino, K., Development of a liquid nitrogen pump using superconducting bulk motor, IEEE Trans. on Applied Superconductivity, Vol. 14, No. 2 (2004), pp. 1659-1662.

Komori, M., Watanabe, K., Kato, H., Asami, K. and Sakai, N., Basic study on active magnetic bearing working in liquid nitrogen and its application to cryogenic pump, Mechanical Engineering Journal, Vol. 4, No. 5 (2017), DOI: 10.1299/mej.16-00723.

Agency for Natural Resources and Energy: Japan's energy 20 Questions to understand the current energy situation (online), available from <https://www.enecho.meti.go.jp/en/category/brochures/pdf/japan_energy_2016.pdf>, (accessed on 7 June, 2019).

Okada, Y., et al., Foundations and applications of magnetic bearings, YouKendo (1995), p. 176 (in Japanese).

Okada, Y., Suzuki, H., Matsuda, K.-I., Kondo, R. and Enokizono, M., Development of Highly Efficient Magnetic Bearing and Application to Ultra-Low Temperature Fluid Pump, Mechanical Engineering Journal, Vol. 2, No. 4 (2015), DOI: 10.1299/mej.15-00086.

P. Wang, Z.B. Fu, T.H. Ding, A frameless eddy current sensor for cryogenic displacement measurement, Sensors and Actuators A : Physical, Vol. 159 , Issue 1 (2010), pp. 7-11.

Sugimoto, H., Shimura, I. and Chiba, A., Design Consideration for Performance Improvement in One-Axis Actively Positioned Single-Drive Bearingless Motor, ISMB15 (2016), pp. 441-445.

Ueno, S. and Arakawa, T., Non-contact rotation test of slotless self-bearing motor, Journal of the Japan Society of Applied Electromagnetics and Mechanics (2011), Vol. 19, No. 2, pp. 407-413 (in Japanese).

Ueno, S. and Okada, Y., Characteristics of Axial Force and Rotating Torque and Their Control of Permanent Magnet Type Axial Gap Self-Bearing Motor, Electrical Engineering in Japan, Vol. 132, No. 1 (2000), pp. 81-91. 